\title{
Relationship Between Cerebral Glucose Metabolic Disorder and Malignant Tumor Type
}

\author{
Siwen Wei ${ }^{\dagger}$, Zhiheng Dong ${ }^{\dagger}$, Ruilian Ma, Sha Li", Rui Cheng* \\ Department of Pharmacy, Affiliated Hospital of Inner Mongolia Medical University, Hohhot, P. R. China \\ Email address: \\ Chengrui237@163.com (Rui Cheng),893477386@qq.com (Sha Li) \\ ${ }^{*}$ Corresponding author \\ $\dagger$ Siwen Wei and Zhiheng Dong are co-first authors.
}

To cite this article:

Siwen Wei, Zhiheng Dong, Ruilian Ma, Sha Li, Rui Cheng. Relationship Between Cerebral Glucose Metabolic Disorder and Malignant Tumor Type. American Journal of Clinical and Experimental Medicine. Vol. 6, No. 4, 2018, pp. 94-98. doi: 10.11648/j.ajcem.20180604.12

Received: May 29, 2018; Accepted: July 20, 2018; Published: August 13, 2018

\begin{abstract}
Aim: To study whether cerebral glucose metabolic disorder in malignant tumor patients without cerebral diseases is related to the site of tumor by using the ${ }^{18} \mathrm{~F}$-deoxyglucose (FDG) PET/CT brain imaging technology. Methods: 22 patients with primary liver cancer and 20 patients with pancreatic cancer were subjected to general physical examinations by ${ }^{18} \mathrm{~F}$-FDG positron emission tomography (PET). A statistical parametric mapping (SPM) software was adopted to analyze the information about cerebral resting glucose metabolism retrospectively, and to compare with the 22 healthy subjects with matched ages and genders. Results: Both the primary liver cancer and pancreatic cancer patients underwent metabolic reduction in both sides of the frontal and temporal areas, but the range and voxel involved in the latter were more extensive than those in the former, especially in the prefrontal cortex. The elevated metabolic areas in pancreatic cancer, which were much wider than those in liver cancer, were mainly located on both sides of the hippocampus, parahippocampal gyrus, amygdala, anterior cingulate cortex and posterior cingulate cortex, and extensively increased glucose metabolism was discerned in cerebellum. The areas of the latter were mainly distributed in the posterior cingulate cortex. Conclusion: Wide areas of glucose metabolic disorder existed in the patients with malignant tumors without cerebral metastasis, the distribution of which is related to the tumor type.
\end{abstract}

Keywords: Cerebral Metabolism, ${ }^{18}$ F-deoxyglucose, Positron Emission Tomography, Psychiatric Oncology

\section{Introduction}

Numerous studies have reported that extensive abnormal areas of resting glucose metabolism exist in the brain of patients with malignant tumors. Subsequent studies have shown that these dysfunctions are significantly associated with the depression levels of cancer patients [1]. The patients with tumors at different parts have different incidence rates of mental and emotional disorders, but it has been unclear that whether the cerebral resting glucose metabolic disorder in patients with malignant tumors is associated with the tumor site or not. Therefore, this study aims to explore the variations of glucose uptake in a resting state of brain in the patients with malignant tumors by comparing the ${ }^{18} \mathrm{~F}$-deoxyglucose (FDG) uptake in the brain of pancreatic and liver cancer patients with that of healthy subjects matched in age, gender and level of education.

\section{Materials and Methods}

\subsection{Subjects}

The retrospective analysis showed that 22 patients with primary liver cancer confirmed by pathology or follow-up included 18 males and 4 females, of whom ages were from 42 to 60 years old and the average age was $[(48.76 \pm 5.43)$ years old], and 20 pancreatic cancer patients consisted of 11 males and 9 females, of whom the oldest was 60 years old, the youngest was 40 years old and the average age was [(47.28 \pm 5.35) years old]. According to the cerebral PET/CT data, after being found occupying lesions by imaging, the patients in the cancer group were subjected to general ${ }^{18} \mathrm{~F}$-FDG-PET/CT examinations in order to determine the nature of disease or preoperative staging, and crania-cerebral organic disease was ruled out through MRI or CT, but the patients accompanied by 
local lymph nodes without distant metastasis were not excluded. 22 healthy subjects were selected as the control in the same period 15 males and 7 females, aged from 41 to 60 years old, with the average age of $(47.13 \pm 6.26)$ years old]. There was no past medical history of epilepsy, cerebral trauma, surgical treatment for brain and cerebrovascular accidents, nor family medical history of mental diseases in the patients and their families in both the cancer group and the control group. The constituent ratios of age, gender and level of education were not significantly different between the two groups $(\mathrm{P}>0.05)$.

\subsection{FDG-PET Imaging}

${ }^{18}$ F-FDG was provided by Copartner System (Shanghai) Co., Ltd. with the radiochemical purity higher than $95 \%$. Cubital intravenous administration was performed according to $5.55 \mathrm{MBq} / \mathrm{kg}$ body weight, after which the patients were required to keep quiet in dark and silent place, and development was conducted after $30 \mathrm{~min}$. A GE Discovery LS PET/CT imager was used to acquire body PET images, and then head PET images in 3D mode. Emission acquisition, and transmission acquisition in which the preset count was 105 and the acquisition time $10 \mathrm{~min}$, were conducted successively. The images were reorganized parallel to the canthomeatal lines with Han filter function and the cutoff frequency of 0.5 . The transverse, coronal and sagittal images were obtained after the reorganization, and the cross-sectional image matrix was $128 \times 128 \times 63$ [2].

\subsection{Statistical Analysis}

After the reorganization, the original images were converted into the Analyze format by MRIcro. SPM2 method was applied to conduct position correction on the images via the Matalab 7.2 platform, and the 16-parameter linear affine transformation and nonlinear iterative method were utilized for unitization processing according to the PET template built in the SPM program. The target image with the matrix of $128 \times 128 \times 63$ and the voxel of $2 \mathrm{~mm} \times 2 \mathrm{~mm} \times 2 \mathrm{~mm}$ was acquired after being smoothed with twice and a half times of width and full height. Two-sample t-test of voxel vs. voxel $(\mathrm{P}<0.001)$ was performed on the ${ }^{18} \mathrm{~F}$-FDG PET images of the patients with liver cancer and pancreatic cancer as well as normal healthy subjects so as to give the distribution graph of cerebral metabolic changes of tumor. The MNI Space utility (MSU) software was used to position pixels with statistically significant differences $(\mathrm{P}<0.001)$, and to calculate the voxel values of glucose metabolic reduction areas and elevated cerebral areas.

\section{Results}

\subsection{Glucose Metabolic Reduction Areas of Liver Cancer and Pancreatic Cancer}

The decline of glucose metabolism in the patients with liver cancer was mainly observed in both sides of the temporal and frontal lobes and anterior cingulate cortex (Table 1, Figure. 1 (1A)-(1C)), in which temporal lobes were mainly distributed in the right middle temporal gyrus and the inferior temporal gyrus, and the left middle temporal gyrus. Frontal lobes in the orbitofrontal cortex (BA 10 and 11 areas on both sides and right BA 47 area), the left anterior cingulate cortex ( BA 32 area) and the right insular cortex and triangularis region (BA 13 and 44 areas).

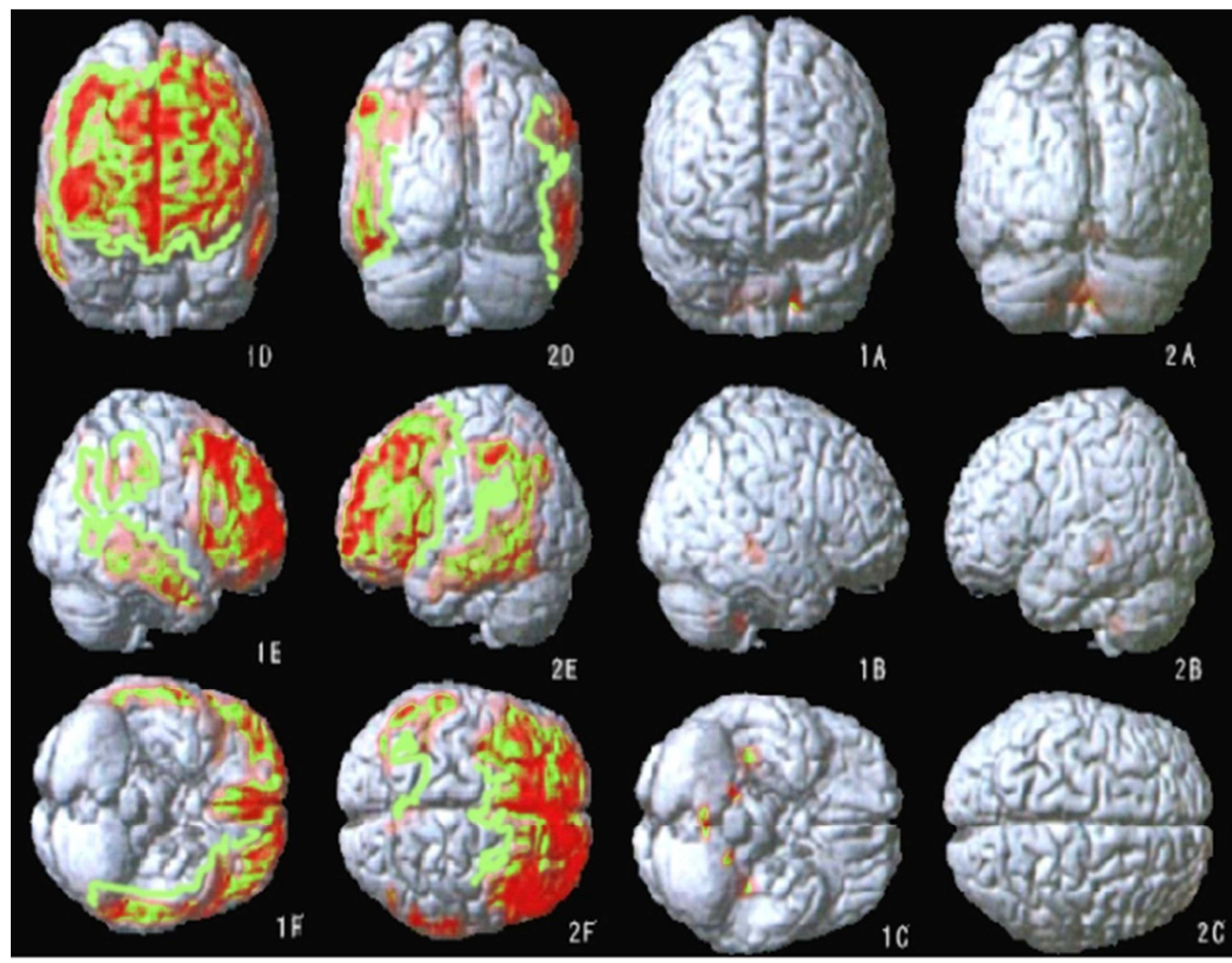

Figure 1. Glucose metabolic elevation areas of liver cancer $(A-C)$ and pancreatic cancer $(D-F)$. 
Table 1. Glucose metabolic reduction areas of liver cancer patients $(P<0.001)$.

\begin{tabular}{|c|c|c|c|c|c|c|}
\hline \multirow{2}{*}{ Cerebral function area } & \multirow{2}{*}{$\begin{array}{l}\text { Brodmann } \\
\text { partition }\end{array}$} & \multicolumn{3}{|c|}{$T$ coordinate } & \multirow{2}{*}{ Voxel No. } & \multirow{2}{*}{$\mathbf{Z}$} \\
\hline & & $\mathbf{X}$ & $\mathbf{Y}$ & $\mathbf{Z}$ & & \\
\hline Superior, middle and inferior temporal gyri (right) & $19,20,37$ & 61 & -9 & -25 & 228 & 3.79 \\
\hline Middle temporal gyrus (left) & 20 & -53 & 5 & -27 & 15 & 3.28 \\
\hline Superior and middle frontal gyri (right) & 9,10 & 33 & 47 & -11 & 16 & 3.12 \\
\hline Superior and middle frontal gyri (left) & 9,10 & -25 & 53 & 0 & 115 & 3.5 \\
\hline Insular cortex, precentral gyrus, inferior frontal gyrus (right) & 12,43 & 47 & 11 & 5 & 61 & 3.62 \\
\hline Superior frontal gyrus, medial frontal lobe, anterior cingulate cortex (left) & $8,9,31$ & -9 & 47 & 30 & 155 & 3.66 \\
\hline Inferior parietal lobule (left) & 2,39 & -51 & -35 & 41 & 53 & 3.62 \\
\hline
\end{tabular}

The areas of glucose metabolic decrease in pancreatic cancer patients were far more extensive than those in liver cancer patients (Table 2, Figure. 1 (1D)-(1F)). These decreased areas were mainly found in both sides of the supplementary motor areas (BA6, 8 and 9 areas), orbital frontal cortex (BA10 and 11 areas), visual association cortex (BA19 area), middle temporal gyrus (BA21 area), inferior temporal gyrus (BA20 area), fusiform gyrus (BA37 area), inferior parietal lobule (BA40 area), angular gyrus (BA39 area), anterior cingulate cortex (BA32 area), auditory cortex (BA42 area), left insular cortex (BA13 area), left primary visual cortex and visual association cortex (BA17 and 18 areas), left superior temporal gyrus (BA22 area) and the right frontal lobe triangularis cortex and pars opercularis cortex (BA44 and 45 areas).

Table 2. Glucose metabolic reduction areas of pancreatic cancer patients $(P<0.001)$.

\begin{tabular}{|c|c|c|c|c|c|c|}
\hline \multirow{2}{*}{ Cerebral function area } & \multirow{2}{*}{$\begin{array}{l}\text { Brodmann } \\
\text { partition }\end{array}$} & \multicolumn{3}{|c|}{ T coordinate } & \multirow{2}{*}{$\begin{array}{l}\text { Voxel } \\
\text { No. }\end{array}$} & \multirow{2}{*}{$\mathbf{Z}$} \\
\hline & & $\mathbf{X}$ & $\mathbf{Y}$ & $\mathbf{Z}$ & & \\
\hline $\begin{array}{l}\text { Superior, middle and inferior temporal gyri, middle occipital gyrus, angular gyrus, } \\
\text { supramarginal gyrus, inferior parietal lobule (left) }\end{array}$ & $\begin{array}{l}18,19,20,21,36 \\
38,41\end{array}$ & -63 & -38 & 3 & 1235 & 4.45 \\
\hline Middle and inferior temporal gyri (right) & $19,20,35$ & 57 & -47 & -15 & 240 & 4.3 \\
\hline Middle and inferior frontal gyri (left) & $5,7,8,9,10,21$ & -30 & 25 & 42 & 4955 & 5.16 \\
\hline Superior, middle and inferior frontal gyri (right) & $5,7,8,9,43,46$ & 58 & 16 & 18 & 3374 & 4.78 \\
\hline Lingual gyrus (both sides), inferior occipital gyrus (left) & 15,16 & -9 & -92 & -15 & 225 & 3.61 \\
\hline Anterior cingulate cortex, superior frontal gyrus, medial frontal lobe (both sides) & $5,8,9,10,31,41$ & -3 & 45 & 16 & 1145 & 4.62 \\
\hline Middle and inferior occipital gyri (right) & 17 & 38 & -87 & -5 & 95 & 3.8 \\
\hline Middle and inferior temporal gyri (right) & 18 & 49 & -62 & -3 & 9 & 3.13 \\
\hline $\begin{array}{l}\text { Superior and middle temporal gyri, angular gyrus, supramarginal gyrus, inferior } \\
\text { parietal lobule (right) }\end{array}$ & 38,41 & 55 & -60 & 27 & 303 & 4.16 \\
\hline Angular gyrus, inferior parietal lobule & 20,40 & -37 & -75 & 33 & 165 & 4.06 \\
\hline Superior frontal gyrus (right) & 10 & 13 & 52 & 35 & 30 & 3.54 \\
\hline Inferior parietal lobule (right) & 38 & 63 & -33 & 45 & 111 & 3.54 \\
\hline Inferior parietal lobule (left) & 38 & -59 & -36 & 41 & 10 & 3.20 \\
\hline
\end{tabular}

\subsection{Glucose Metabolic Elevation Areas of Liver Cancer and Pancreatic Cancer}

posterior cingulate cortex, and the right side in particular, followed by the primary somatosensory cortex area of postcentral gyrus (Table 3, Figure. 1 (2A)-(2C)).

A small amount of elevated metabolic areas existed in the patients with liver cancer, which were concentrated in the

Table 3. Glucose metabolic elevation areas of liver cancer $(P<0.001)$

\begin{tabular}{|c|c|c|c|c|c|c|}
\hline \multirow{2}{*}{ Cerebral function area } & \multirow{2}{*}{ Brodmann partition } & \multicolumn{3}{|c|}{ T coordinate } & \multirow{2}{*}{ Voxel No. } & \multirow{2}{*}{$\mathbf{Z}$} \\
\hline & & $\mathbf{X}$ & $\mathbf{Y}$ & $\mathbf{Z}$ & & \\
\hline Cuneate lobe, posterior cingulate cortex (both sides) & $19,32,33$ & 0 & -68 & 11 & 106 & 3.39 \\
\hline Postcentral gyrus (right) & 4 & 25 & -35 & 57 & 5 & 3.12 \\
\hline
\end{tabular}

Table 4. Glucose metabolic elevation areas of pancreatic cancer $(P<0.05)$.

\begin{tabular}{|c|c|c|c|c|c|c|}
\hline \multirow{2}{*}{ Cerebral function area } & \multirow{2}{*}{ Brodmann partition } & \multicolumn{3}{|c|}{$T$ coordinate } & \multirow{2}{*}{ Voxel No. } & \multirow{2}{*}{$\mathbf{Z}$} \\
\hline & & $\mathbf{X}$ & $\mathbf{Y}$ & $\mathbf{Z}$ & & \\
\hline Cerebellum (both sides) & & -20 & -45 & -45 & 3055 & 4.29 \\
\hline $\begin{array}{l}\text { Posterior cingulate cortex, fusiform gyrus, parahippocampal gyrus, } \\
\text { thalamus, caudate nucleus, hippocampus, amygdala (right) }\end{array}$ & $\begin{array}{l}12,18,28,20,21,26 \\
28,29,35,38\end{array}$ & 36 & -20 & -15 & 1393 & 4.87 \\
\hline $\begin{array}{l}\text { Parahippocampal gyrus, fusiform gyrus, thalamus, caudate nucleus, } \\
\text { hippocampus, amygdala (left) }\end{array}$ & $\begin{array}{l}18,19,26,27,29,33 \\
34,35,36\end{array}$ & -20 & -5 & -18 & 1101 & 4.59 \\
\hline Anterior cingulate cortex (left) & $10,24,30$ & -7 & 23 & -11 & 53 & 3.65 \\
\hline Insular cortex (right) & 12 & 25 & 11 & 17 & 151 & 4.06 \\
\hline Putamen (left) & & -22 & 11 & 17 & 132 & 3.73 \\
\hline Posterior cingulate cortex (left) & 30 & -15 & -38 & 32 & 22 & 3.79 \\
\hline
\end{tabular}


The elevated areas of cerebral resting glucose metabolism were more extensive in the patients with pancreatic cancer than in those with liver cancer (Table 4, Figure. 1 (2D)-(2F)), which mainly included the both sides of the cerebellum, both sides of the hippocampus, parahippocampal gyrus, amygdala, anterior cingulate (BA25 area) and posterior cingulate. The metabolisms of the right insular cortex, left orbitofrontal area (BA11 area) and parolfactory cortex, both sides of thalamus and caudate nucleus were higher than those of the normal control group.

The metabolism of both sides of the frontal and temporal areas of the two tumors declined, and the decreased areas of glucose metabolism in the patients with pancreatic cancer were more extensive than that in pancreatic cancer patients. Especially in terms of the comparison in the elevated areas of glucose metabolism between liver cancer and pancreatic cancer shown in Figure. $1(2 \mathrm{~A})-(2 \mathrm{~F})$, the elevated area of the latter was significantly larger than that of the former. The elevated areas of glucose metabolism were mainly concentrated in the posterior cingulate cortex in the patients with liver cancer on the right side in particular, and mainly located on both sides of hippocampus, parahippocampal gyrus, amygdala, anterior cingulate cortex and posterior cingulate cortex in the patients with pancreatic cancer. In the meantime, glucose metabolism in cerebellum was also evidently elevated.

\section{Discussion}

PET/CT has been widely used in the diagnosis, staging and recurrence judgment of malignant tumors. In recent years, some scholars have begun to study resting glucose metabolism of tumor patients by PET and taken the subject of tumor psychiatry into the research field of imaging, and also created a new area for the application of PET. Many previous studies have found that metabolic abnormalities of the limbic system and marginal side structure exist in the brain of patients with malignant cancer, which is mainly in the left frontal lobe. There are many similarities between these manifestations and local cerebral glucose metabolic changes of primary depression [3].

This study also showed that all tumors at different parts could be found similar abnormal glucose metabolism mainly in frontal and temporal lobes, but the metabolic abnormalities of pancreatic cancer had more extensive distribution than that of liver cancer, which had not yet been reported in previous studies. Literatures have reported [4] that there are about half of cancer patients suffering from a variety of mental disorders at the same time, the most common of which is adjustment disorder and severe depression in advanced tumors. The morbidity of emotional disorder and the severity of symptoms are different in tumors with different pathological types at different parts. The tumor psychiatry survey have shown that the incidence of depression of pancreatic cancer patients is much higher than that of patients with liver cancer, which is up to about $35 \%$ to $55 \%$, and the incidence of depression is the lowest in patients with gastric cancer $(9 \%)$ and leukemia $(1.6 \%)$. Pancreatic cancer is the most malignant tumor with neuroendocrine functions, which often enters a later period when found, and its five-year survival rate is only $6 \%$. Autoimmune response of tumor, tumorous malnutrition and endocrine dysfunction can also cause remote effects of central nervous system. In fact, the incidence of depression and suicide is also the highest in pancreatic cancer patients. Some scholars believe that it may be related to endocrine changes caused by pancreatic tumor.

In addition, the treatment of cancer may have varying levels of impact on the central nervous system, such as the neurotoxic effects of chemotherapy drugs, and central analgesic drugs.

This study also found the areas which existed increased cerebral glucose metabolism in cancer patients were mainly in the hippocampus, parahippocampal gyrus, cingulate gyrus and posterior cingulate gyrus in particular, and pancreatic cancer was also accompanied by elevated amygdala metabolism. There were no similar reports in previous studies [5]. When scenario stimulation was performed on patients with post-traumatic stress disorder (PTSD), the results showed hyperactivity took place in amygdala, parahippocampal gyrus, insular cortex, inferior parietal lobule, cingulate gyrus and precuneus [6]. Different from anxiety disorder, under-action was only found in PTSD, including the inferior parietal lobule, ventromedial prefrontal cortex, cortex, parahippocampal gyrus, lingual gyrus, dorsal amygdala, anterior edge of hippocampus, orbitofrontal cortex, putamen, middle occipital gyrus, dorsomedial prefrontal cortex, dorsal anterior cingulate cortex and middle cingulate cortex, in which the activity of medial PFC was inversely proportional to the severity of the symptoms of PTSD [7]. The functions of anterior edge of hippocampus were relative with declarative memory and the functional regulation of hypothalamus - pituitary - adrenal axis. Both of the functions were disordered in PTSD patients, which were also related with endogenous anxiety [8]. In view of a most common condition that PTSD is combined with depression, therefore, PTSD pathophysiological changes may exist in pancreatic cancer patients. In fact, Inagaki et al. [9] also found that the scores of depressive patients were also significantly higher than those of non-depressed patients in the Impact of Event Scale (Revised). This may be a unique pathophysiological change of cancer secondary depression.

The data showed that the metabolism of below-knee anterior cingulate cortex (sACC, BA25 area) of pancreatic cancer patients elevated, which was the same as the report of Inagaki et al. [9] who also found that there was significant correlation between the local sACC glucose metabolic rate and the score of Hamilton Depression Scale. Bi-directional fibrous connection existed between SACC and orbitofrontal cortex, hypothalamus, amygdala and among nucleus accumbens, and these structures were related to emotional behaviors. sACC hyperthyroidism may be associated with the fact that pancreatic cancer is more vulnerable to secondary depression. Unlike cancer-related depression, the 
neuroimaging studies on primary depression showed that the glucose metabolism of sACC declined [10]; the blood flow and metabolism of below-knee prefrontal cortex (BA24 and 25 areas) slowed down in patients with unipolar depression and bipolar depression, and this change had the tendency of left lateralization. Cortical atrophy in the area was also found by MRI. If the impact caused by gray-matter volume reduction is corrected, it will be found that the metabolism of the residual below-knee prefrontal cortex is elevated. After effective treatment, the hyperfunctional areas can be restored to their normal state [11]. Hyperfunction can also be found in the area when performing sadness simulation on normal persons without depression. In the previous studies on brain function imaging, cerebellum was often regarded as a reference region, for many studies suggest that cerebellum is mainly involved in the coordination of movement, and blood flow or metabolism of cerebellum is relatively spared in patients with functional mental disorder. The imaging studies in recent years have shown that cerebellar metabolism may also change in other nerves and nerve disorders, including memory disorders. Cerebellum has a certain effect in cognitive impairment in patients with depression, which may involve some subtypes of depression. The data also showed that the areas where glucose metabolism was elevated also existed in the cerebellum of the patients. At present, evidence has been mounting that cerebellum is also involved in the processing of human emotions. Bench et al. [12] reported that the patients with heavy unipolar depression experienced increased cerebellar vermis blood flow. There were also researches suggesting that the cerebellar language and cognition is related to negative emotions. In patients with depression accompanied by cognitive impairment, the decline of metabolic levels is more severe than that in those not associated with cognitive impairment, and their activity of cerebellar vermis increased. The MRI studies of Shah et al. [13] showed that the cerebellar structures of patients with severe depression changed abnormally.

\section{Conclusion}

In short, this study showed that extensive reduced metabolic areas existed in the brains of patients with cancers at different parts, mainly in the frontal and temporal lobes, and the distributions of cerebral metabolic abnormalities were different in the tumors at different parts, which suggest that the influence of the location of tumor on cerebral functions must be considered in further studies in the future.

\section{Acknowledgements}

This research is funded by the following projects: the Major scientific research projects of Affiliated Hospital of Inner Mongolia Medical University (NYFY ZD 2012017); the
Nature Science Foundation of Inner Mongolia Autonomous Region (2017MS0831 and 2018LH08032), http://nsbr.nmkjt.gov.cn/index.aspx

\section{References}

[1] Betul O, Ipek M. Brain tumor presenting with psychiatric symptoms. J Neuropsychiatry Clin Neurosci 2011;23:43-44.

[2] Ni JM, Lin MF, Liu JJ, Huang G. [Regional brain metabolism changes in the body malignant tumor patients without brain metastasis]. Chin J Med Imaging Technol 2010;26:2175-2177.

[3] Gulyás B, Halldin C. New PET radiopharmaceuticals beyond FDG for brain tumor imaging. Q J Nucl Med Mol Imaging 2012;56:173-190.

[4] Ozeki Y, Abe Y, Kita H, Tamura K, Sakata I, Ishida J, Machida $\mathrm{K}$. A case of primary lung cancer lesion demonstrated by F-18 FDG positron emission tomography/computed tomography (PET/CT) one year after the detection of metastatic brain tumor Oncol Lett 2011;2:621-623.

[5] Vermetten E, Lanius RA. Biological and clinical framework for posttraumatic stress disorder. Handb Clin Neurol 2012;10:291-342.

[6] Javidi H, Yadollahie M. Post-traumatic Stress Disorder. Int J Occup Environ Med 2012;3:2-9.

[7] Fujita Y, Yamamoto S, Morinobu S. Novel therapeutic approach for the treatment of post-traumatic stress disorder (PTSD): facilitating fear extinction. Nihon Shinkei Seishin Yakurigaku Zasshi 2012;32:195-201.

[8] Acheson DT, Gresack JE, Risbrough VB. Hippocampal dysfunction effects on context memory: possible etiology for posttraumatic stress disorder. Neuropharmacology 2012;62:674-685.

[9] Inagaki M, Yoshikawa E, Kobayakawa M, Matsuoka Y, Sugawara Y, Nakano T, Akizuki N, Fujimori M, Akechi T, Kinoshita T, Furuse J, Murakami K, Uchitomi Y. Regional cerebral glucose metabolism in patients with secondary depressive episodes after fatalpancreatic cancer diagnosis. J Affect Disord 2007;99:231-236.

[10] Puskás T, Henits I. Diffusion-weighted MR imaging; the significance of $\mathrm{ADC}$ and perfusion values in the differential diagnosis of pancreatic adenocarcinoma and mass forming pancreatitis. Orv Hetil 2012;153:1191-1196.

[11] Drevets WC. Functional anatomical abnormalities in limbic and prefrontal cortical structures in major depression. Prog Brain Res 2000;126:413-431.

[12] Bench CJ, Friston KJ, Brown RG, Scott LC, Frackowiak RS, Dolan RJ. The anatomy of melancholia--focal abnormalities of cerebral blood flow in major depression. Psychol Med 1992;22:607-615.

[13] Shah PJ, Glabus MF, Goodwin GM, Ebmeier KP. Chronic, treatment-resistant depression and right fronto-striatal atrophy. Br J Psychiatry 2002;5:430-440. 